\title{
Relação entre Reservas Orçamentárias e Orientação de Valor Social
}

\author{
Maria Carolina Gazso Righetti ${ }^{1}$ \\ Ricardo Lopes Cardoso ${ }^{2}$ \\ José Carlos Tiomatsu Oyadomari ${ }^{3}$ \\ Octávio Ribeiro de Mendonça Neto ${ }^{4}$
}

\section{Resumo}

Os estudos sobre reservas orçamentárias ainda continuam inconclusivos, mas destaque tem sido dado aos aspectos comportamentais. O presente trabalho inova ao testar empiricamente se a orientação de valor social (comportamento pró-social, competitivo e individual) dos indivíduos pode contribuir para a formação de folga orçamentária. $\mathrm{O}$ estudo é do tipo single entity, com a pesquisa sendo feita com 41 gestores de uma multinacional americana do segmento de autopeças. A pesquisa foi realizada em duas fases: Na primeira, os participantes responderam a um instrumento de orientação de valor social. E na segunda fase do procedimento, os participantes foram submetidos a um experimento orçamentário a fim de se analisar o processo de formação de folga orçamentária. Os resultados confirmaram a propensão para formação de folga orçamentária, mas não foi possível confirmar as hipóteses teóricas de que a orientação de valor social cooperativa contribui para a redução da folga orçamentária, sugerindo que outros fatores possam estar interferindo nesse fenômeno.

Palavras-chave: Orçamento; Folga Orçamentária; Teoria de Orientação de Valor Social.

\section{Relationship between Budgetary Slack and Social Value Orientation}

\begin{abstract}
Studies regarding budget slack are still inconclusive, however, emphasis has been given to the behavioral aspects. This present study innovates by testing empirically if if social value orientation (prosocial competitive and individual behavior) of the individuals can contribute to budgetary slack generation. This is a sing entity study, where the research was conducted with 41 managers of an American multinational of automotive parts segment. The research was conducted in two phases: In the first phase participants answered a questionnaire on social value orientation. And in the second phase, participants were submitted to a budget experiment in order to analyze the formation budgetary slack. Based on the statistical results, it was not possible to confirm the theoretical hypotheses that the cooperative social value orientation helps to reduce the budgetary slack, suggesting that others factors might be interfering in this phenomenon.
\end{abstract}

Keywords: Budget; Budgetary Slack; Social Value Orientation.

\footnotetext{
${ }^{1}$ Professora Universidade Presbiteriana Mackenzie. righetti.carolina@gmail.com

${ }^{2}$ FIPECAFI. ricardocardoso123@gmail.com

${ }^{3}$ Professor do Curso de Mestrado Profissional do Programa de Pós Graduação em Ciências Contábeis do Centro de Ciências Sociais Aplicadas - CCSA da Universidade Presbiteriana Mackenzie. oyadomari@mackenzie.br

${ }^{4}$ Universidade Presbiteriana Mackenzie. octavio.mendonca@mackenzie.br
} 


\section{Introdução}

Apesar do orçamento ser uma das ferramentas de controle gerencial mais tradicionais e ser abundante em termos de quantidade de pesquisas, nota-se que ainda este tema continua relevante no ambiente de pesquisas e no ambiente da prática. De um lado há grande crítica sobre o papel deste artefato, como preconizado pela filosofia de gestão denominada Beyond Budgeting (Hope \& Fraser, 2003), que tem como proposta resolver os principais problemas do orçamento, como comportamentos disfuncionais, alto gasto de tempo e recursos, falta de atualização e de não estar orientado para o cliente (HENTTU-AHO \& JÄRVINEN, 2013), mas mesmo com críticas ao orçamento há que se considerar os resultados apontados por Libby \& Lindsay (2010) com empresas canadenses e norte-americanas, os quais mostram o orçamento continua sendo valioso e pode continuar sendo útil, desde que feito pequenos ajustes vinculados às áreas de recursos humanos e visão de desempenho de longo prazo.

Um dos problemas apontados na literatura é a reserva orçamentária (budgetary slack), a qual deriva-se dos estudos relacionados com a folga organizacional (CYERT e MARCH, 1992). A reserva orçamentária ocorre quando o indivíduo superestima despesas e custos ou subavalia receitas no intuito de facilitar o alcance de suas próprias metas. Esse tipo de comportamento afeta negativamente os processos de planejamento e orçamento devido aos vieses introduzidos na alocação ou na utilização de recursos (MERCHANT, 1985). Esse construto junto com outros construtos relatados na literatura como miopia gerencial (MERCHANT, 1990), rigidez orçamentária e visão de curto prazo (VAN DER STEDE, 2000), podem ser caracterizados como problemas do orçamento (FREZATTI et al., 2010), talvez os principais, já que carregam potenciais efeitos negativos no desempenho da empresa.

Além da questão do desempenho, em geral a alocação inadequada de recursos pode causar problemas no processo de controle gerencial pelos seguintes motivos: (i) as pessoas que estão mais perto das operações podem possuir informações privilegiadas e, com isso, favorecer a si próprias, com o agravante de que o custo de monitorar diretamente essas pessoas na maioria das vezes se torna inviável; (ii) os profissionais possuem recursos de conhecimento limitados e não podem ser responsabilizados por falha nos projetos (por exemplo, tendo de cobrir com seus próprios recursos se houver um eventual prejuízo); (iii) por outro lado a folga orçamentária é vista como desejável por parte dos profissionais por trazer flexibilidade ao trabalho deles (SCHWARTZ et al., 2007), o que, do ponto de vista de avaliação de desempenho, poderia favorecer os profissionais que adotam esse tipo de procedimento em detrimento de outros que não o adotam . 
Em uma tentativa de quantificar esse problema, Schiff e Lewin (1968 como citado em SCHWARTZ et al., 2007) estimam que a folga orçamentária seja responsável por cerca de 20 a 30 por cento das despesas divisionais, o que qualifica essa atitude como um problema relevante em termos de representatividade de recursos.

As condições descritas sugerem que os subordinados têm oportunidade e motivação para agir de forma não alinhada com os seus superiores e com as metas organizacionais produzindo danos à organização, seguindo interesses próprios. Mas ao contrário do que sugerem as teorias tradicionais, como a Teoria da Agência, que pressupõem este princípio de autointeresse racional (ou homem econômico), Van Lange, et al., (1997) afirmam que desenvolvimentos teóricos mais recentes têm indicado que os indivíduos diferem sistematicamente na maneira em que se relacionam entre si em uma situação de interdependência. Segundo McClintock e Messick (1969), algumas pessoas estão inclinadas a agir de forma mais cooperativa que as outras, e essas diferenças podem estar relacionadas com bases motivacionais de cooperação e competição.

Dessa forma, uma linha de pesquisa que pode servir como alternativa à Teoria da Agência são os estudos sobre Orientação de Valor Social. Os estudos sobre a orientação de valor social e seus efeitos sobre o comportamento das pessoas em relação aos seus pares vêm abrindo novos horizontes para a compreensão do comportamento humano, o que pode ser valioso para a área de Contabilidade Gerencial, considerando-se que as organizações dependem cada vez mais do desempenho de seus grupos de funcionários para atingir seus objetivos (UPTON, 2009).

Nos estudos sobre orientação de valor social, há uma proposta de classificação sobre os três tipos de comportamento: (i) pró-sociais (que valorizam a cooperação e a igualdade nos resultados); (ii) individualistas (que se concentram em suas próprias recompensas); e (iii) concorrentes (que buscam vantagem relativa sobre os outros) (VAN LANGE et al.,1997).

Assim o comportamento pró-social tem implicações importantes para as organizações (BRIEF e MOTOWIDLO, 1986), uma vez que descreve os padrões de comportamento considerados necessários para a eficácia do funcionamento organizacional. Por comportamento pró-social, pode-se entender o que vai além da função exigida - como cooperar com os colegas de trabalho; agir, quando necessário, para proteger a organização do perigo inesperado; sugerir formas de melhorar a organização; promover o autodesenvolvimento; preparar-se para níveis mais elevados de responsabilidade organizacional e falar positivamente sobre a organização para outras pessoas. 
Segundo Brief e Motowidlo (1986), durante os anos 1960 e início de 1970, inúmeros cientistas comportamentais e sociais se dedicaram a estudar os comportamentos pró-sociais, porém com poucos estudos voltados para as organizações.

Fundamentado nos argumentos relatados e na busca de compreender as causas e os efeitos de aspectos comportamentais envolvidos no processo orçamentário definiu-se a seguinte questão de pesquisa como sendo: Qual é a relação entre orientação de valor social e folgas orçamentárias?

O objetivo desta pesquisa é contribuir para o avanço nos conhecimentos acerca da contabilidade gerencial. Neste contexto, o processo orçamentário é um componente importante no sistema de medição de desempenho das organizações. Estes sistemas tem sido foco de diversos estudos nas últimas décadas, mas ainda não são conclusivos e são poucos os trabalhos que se dedicaram a compreender como o comportamento dos indivíduos influenciam o funcionamento destes sistemas (SANTOS, LUCIANETTI e BOURNE, 2012 pág. 99).

Vale lembrar que a maneira como se julga a escolha mais apropriada depende, em algum grau, do sistema de valores que cada indivíduo guarda e que funciona como um princípio norteador do seu curso de ação preferencial. Por este motivo, o conceito de valor ocupa papel central nos diversos campos de estudo focados no comportamento humano (IWAI, 2014 pág. 2). Considerando os altos custos de implementação e manutenção é fundamental que os profissionais de controladoria entendam quais as melhores práticas para que esses sistemas funcionem de forma eficaz.

A importância desta pesquisa se justifica pelo alto custo de implementação e manutenção dos sistemas de medição de desempenho, os quais por si só não garantem melhora de desempenho para as organizações. A partir deste estudo, os profissionais de controladoria podem entender melhor a respeito de como as pessoas respondem aos sistemas de medição de desempenho, quais as melhores ferramentas para a realização destes trabalhos de maneira eficaz para as organizações.

Dadas as considerações acima, o presente artigo apresenta a seguinte estrutura. $\mathrm{Na}$ sequencia apresenta-se o referencial teórico sobre orientação de valor social e reservas orçamentárias. Com base nestes conceitos as hipóteses são apresentadas e justificadas A seguir, apresenta-se os procedimentos metodológicos onde é detalhado o estudo de caso bem como os instrumentos utilizados para mensurar a orientação de valor social e folga orçamentária. Depois disto, os resultados são apresentados e discutidos. E finalmente, as principais contribuições do estudo, bem como suas limitações, são levantadas na seção final. 


\section{Referencial Teórico}

Esta seção abrange os dois temas que fundamentam esse artigo: Reservas Orçamentárias e Orientação de Valor Social e é a base para justificar as hipóteses.

\subsection{Orientações de valor social}

Embora uma variedade de diferentes orientações de valor social possa ser distinguida de um ponto de vista teórico (VAN LANGE et al., 1997), no presente trabalho são discutidas três categorias de orientação de valor social: pró-social, individualista e competitiva.

Indivíduos com orientação de valor pró-social tendem a maximizar os resultados tanto para si próprios quanto para os outros em uma postura de cooperação, além de minimizar as diferenças entre os resultados para si e para os outros em uma postura de igualdade; já os individualistas tendem a maximizar seus próprios resultados com pouca ou nenhuma consideração com relação aos outros resultados - enquanto os concorrentes ou os competidores tendem a maximizar seus próprios resultados relativos aos resultados dos outros, buscando vantagem relativa sobre os outros (VAN LANGE et al., 1997).

Mais especificamente sobre os indivíduos com orientação de valor pró-social, Brief e Motowidlo (1986), embora reconhecendo que não exista consenso estabelecido sobre uma definição precisa de comportamentos pró-sociais, sugerem o raciocínio seguinte: o comportamento pró-social é um comportamento organizacional que é (a) realizado por um membro de uma organização, (b) dirigido a um indivíduo, grupo ou organização com quem ele ou ela interage enquanto exerce sua função na organização, e (c) realizado com a intenção de promover o bem-estar do indivíduo, do grupo ou da organização para a qual se dirige. Essa definição abrange uma ampla gama de comportamentos que têm em comum, como noção central, a intenção de beneficiar os outros.

Existem várias diferenças importantes entre os tipos de comportamento organizacional pró-social. Primeiramente, alguns comportamentos pró-sociais são funcionais, pois contribuem para a realização dos objetivos organizacionais e são necessários para o funcionamento da organização, enquanto outros são disfuncionais (BRIEF e MOTOWIDLO, 1986).

A organização tem mais chances de prosperar quando seus membros cooperam entre si, agem para protegê-la contra os riscos e falam favoravelmente sobre ela para os outros etc., melhorando a capacidade da organização de sobreviver e de alcançar seus objetivos. Atos 
como ajudar, partilhar e cooperar possuem legítimas dimensões no desempenho do trabalho. Organ (1977 como citado em BRIEF e MOTOWIDLO, 1986) argumentou que comportamentos como esses muitas vezes são considerados pelos gestores como ainda mais importantes do que uma excepcional produtividade.

Por outro lado, cabe destacar que há outras expressões de comportamentos pró-sociais que podem diminuir a eficácia organizacional. Membros da organização que ajudam os colegas de trabalho a atingir metas pessoais que não estão em conformidade com os objetivos organizacionais, que deliberadamente falsificam registros para proteger os outros da censura organizacional ou que prestam serviços a clientes de forma contrária aos interesses organizacionais, por exemplo, estão realizando atos pró-sociais que não contribuem para o sucesso organizacional (BRIEF e MOTOWIDLO, 1986).

Há ainda outro aspecto importante que envolve os alvos aos quais os atos pró-sociais são direcionados. Muitas vezes, os alvos são pessoas com as quais os membros organizacionais interagem, e os atos pró-sociais são realizados com a intenção de beneficiar esse alvo diretamente. O alvo pode ser um colega de trabalho (ou seja, supervisor, par ou subordinado) ou um cliente da organização.

Staw (1983, 1984 como citado em BRIEF e MOTOWIDLO, 1986, p. 711), entretanto, argumenta que o comportamento pró-social não precisa ser restrito a ações tendo como alvo indivíduos podendo incluir ações direcionadas para a organização. Este autor descreveu como atos pró-sociais organizacionais o serviço voluntário para a organização, a cooperação com os procedimentos organizacionais, o esforço extra para com os objetivos organizacionais e as expressões de lealdade. Isso significa que os indivíduos estariam doando tempo, energia e outros recursos pessoais e, nesse sentido, sacrificando alguma parte de seus próprios interesses para o bem da organização. Embora os atos pró-sociais com alvo nos indivíduos possam ser funcionais ou disfuncionais para a organização, os atos pró-sociais com alvo na organização são quase sempre funcionais (BRIEF e MOTOWIDLO, 1986).

\subsection{Reservas orçamentárias}

Quanto às Reservas Orçamentárias, se forem analisadas dentro de uma perspectiva que enxerga aqueles comportamentos de forma negativa para a organização, ocorrem quando o gestor, sem o consentimento de seus superiores: (1) superestima os gastos, (2) subestima as receitas e (3) superestima a demanda de recursos sob sua responsabilidade. O objetivo do gestor ao constituir essa reserva é proteger-se objetivando ter uma avaliação de desempenho 
que o favoreça, priorizando os seus interesses em detrimento dos da organização, em um claro problema de conflito de agência (JUNQUEIRA et al., 2010).

A teoria da agência trata da relação de agência (designada como um contrato), segundo a qual uma parte (o principal) delega uma determinada tarefa para outra parte (o agente), que realiza tal tarefa. A teoria da agência está focada em solucionar dois problemas que podem ocorrer nessa relação. $O$ primeiro seria o conflito de interesses entre o principal e o agente e a dificuldade (custo) que o principal tem para verificar se as ações do agente são apropriadas. O segundo acontece quando o principal e o agente têm visões diferentes em relação ao risco (EISENHARDT, 1989).

Desse conflito de interesses pode surgir a formação de folga orçamentária, a qual representa um problema de planejamento e alocação de recursos para as empresas. Quanto a isso, Oliveira et al. (2010) afirmam que a folga orçamentária surge de forma natural quando os valores estimados em orçamento não são objeto de uma reflexão mais profunda, como, por exemplo, a simples utilização de registros anteriores. Dessa forma, a folga é construída com o objetivo de criar gordura, afetando negativamente os processos de planejamento, mediante a alocação de recursos em desacordo com as necessidades da organização. Ou seja, pela concepção de eficiência e eficácia organizacional, a folga orçamentária representa o desajuste das metas da organização e configura-se em um fenômeno a ser inibido, o que requer o conhecimento de suas características, motivações e reflexos (BEUREN, BECK e POPIK 2014).

Em suma, os trabalhos que se preocupam com as causas da constituição da folga orçamentária não são conclusivos, mas seus achados podem contribuir para o maior entendimento do assunto e para futuros progressos acerca desse problema. Os principais pontos levantados pelos autores sobre as possíveis causas de constituição de folga orçamentária são: (1) Estilo de avaliação de desempenho; (2) Comprometimento organizacional; (3) Participação no orçamento; (4) Imposição das Metas; e (5) Sistema de informação e avaliação de desempenho (Junqueira et al., 2010)

Com base nessas considerações, declara-se a Hipótese H1: Existe propensão à criação de folgas orçamentárias em ambientes nos quais o estilo de avaliação de desempenho é baseado no cumprimento do orçamento.

Mas, conforme já discutido, o grau de comprometimento organizacional dos funcionários é um componente importante. Alguns fatores que podem contribuir para o comprometimento são (i) aceitação de metas, processo de feedback com relação ao cumprimento ou não das metas e (ii) metas claras e desafiadoras, mas exequíveis (Junqueira

ReFAE - Revista da Faculdade de Administração e Economia, v. 9, n. 1, p. 117-134, 2018 
et al., 2010).

Esses fatores podem estar relacionados com o conceito de orientação de valor social e, mais precisamente, com o comportamento pró-social. Ou seja, os fatores citados como motivadores do comprometimento organizacional - aceitação de metas, processo de feedback construtivo e bom relacionamento entre pares, entre subordinados e entre subordinados e superiores - são características de indivíduos pró-sociais, que percebem seus objetivos de forma cooperativa, o que, por sua vez, pode estar associado à redução da folga orçamentária.

Com base nesses pressupostos teóricos, declara-se a Hipótese H2: Existe diferença na formação de folga orçamentária entre indivíduos com diferentes tipos de orientação social.

\section{Procedimentos Metodológicos}

\subsection{Classificação da pesquisa}

Quanto à abordagem, esta pesquisa se classifica como qualitativa, pois procura aprofundar a compreensão de processos dinâmicos vividos por grupos sociais, possibilitando o maior entendimento das particularidades do comportamento dos indivíduos. Mais especificamente, procura compreender se a orientação de valor social dos indivíduos (comportamento pró-social, competitivo e individual) pode contribuir para a formação de folga orçamentária.

Quanto ao objetivo geral, esta pesquisa pode ser classificada como exploratória, já que tem como objetivo proporcionar maior familiaridade com o problema orçamento, porém integrando com o fenômeno da folga orçamentária e relacionando com tipos de orientação social. O objetivo da pesquisa exploratória é tornar o problema mais explícito ou ainda construir hipóteses envolvendo análise de exemplos que estimulem sua compreensão sobre pesquisas já desenvolvidas (DIEHL e TATIM, 2004).

Por fim, quanto ao procedimento técnico, esta pesquisa se utilizou do estudo de caso único. Segundo Diehl e Tatim (2004), o estudo de caso caracteriza-se pelo estudo profundo e exaustivo de um ou poucos objetos (neste caso, gestores de uma empresa denominada Tower Automotive), de maneira que permita seu amplo conhecimento.

\subsection{Objeto de estudo}

A Tower International é uma multinacional americana que desenvolve e produz componentes metálicos para a maioria de montadoras de veículos. Com 40 filiais em 13 
países, possui cerca de 8.000 funcionários. O grupo, presente no Brasil por meio da Tower Automotive do Brasil (com filiais em Arujá, Betim e Contagem), iniciou suas atividades quando adquiriu, em 1997, o que era originalmente uma empresa familiar. Seus principais clientes no Brasil são Volkswagen, Fiat, Peugeot e Honda.

Em relação ao sistema de avaliação, a Tower é guiada principalmente pelo Ebitda (Earnings before taxes, depreciation and amortization), seguido das medidas de desempenho como geração de caixa, ganho de novos negócios, melhoria de margem, performance de qualidade, segurança e controles internos (avaliados por auditorias interna e externa).

A área de Controladoria é responsável por coordenar o processo orçamentário que acontece anualmente, com início em meados de setembro e término em dezembro. $\mathrm{O}$ controller juntamente com sua equipe elabora um cronograma detalhado de todo o processo que consiste basicamente em coletar informações internas (ganho de novos negócios, metas definidas pela diretoria, plano de melhoria contínua, investimento em novos equipamentos) e informações externas (informações de mercado, bancos e governo acerca de crescimento do setor, níveis de inflação, limite de crédito, medidas de incentivo governamentais, negociações sindicais, entre outros), elaborar as projeções detalhadamente no formato de demonstrativo de resultado e fluxo de caixa (para o período de um ano, (subsequente á sua elaboração), subdividido em meses), avaliar, emitir parecer técnico e discutir com a diretoria sobre o nível de riscos e oportunidades destas projeções comparando com o cenário real/atual. E finalmente, comunicar a conclusão dos trabalhos, divulgando o orçamento oficial para a Companhia e registrando o mesmo em sistema corporativo (formato padronizado de relatórios). É importante citar que todos os passos são realizados através de reuniões de trabalho lideradas pela equipe da Controladoria com a participação da diretoria/presidência local e diretoria da matriz). Estas reuniões são formalizadas através de lista de presença e atas que contemplam as tomadas de decisões).

Com base nesse material, a Controladoria é incumbida de subdividir as metas orçamentárias e distribuí-las aos gestores de todas as áreas (exemplo: vendas em unidades e preço unitário para a área comercial, despesas de manutenção e quantidade de operários para área operacional e assim por diante. Adicionalmente, a Controladoria elabora mensalmente novas projeções, (forecast), baseando-se em informações atualizadas de forma mais compacta ao trabalho orçamentário e avalia o desempenho da empresa, comparando o real/forecast com o orçamento. Este sistema de avaliação também é subdividido por gestor, para facilitar o entendimento dos resultados e a elaboração de plano de recuperação com os devidos responsáveis. 
O desempenho dos gestores influencia diretamente no esquema de remuneração da empresa. Este consiste em salário fixo mais um bônus variável. O bônus é pago anualmente com base no cumprimento das metas estipuladas - a de maior peso nesse cálculo é o Ebitda, que está diretamente ligado ao cumprimento do orçamento dos gestores e a pressão por resultados.

Desta forma, fica evidente, que a Tower Automotive tem um sistema robusto de controle gerencial e que a área de Controladoria tem papel fundamental na condução deste sistema. Este papel se torna mais desafiador levando-se em consideração a dinâmica do setor automotivo no Brasil e no mundo. De acordo com Costa e Queiroz (2000) desde o final dos anos 70, com o aumento da competição provocado pelos veículos japoneses de alta qualidade, desempenho e segurança e com a elevação do nível de exigência dos consumidores as montadoras ocidentais passaram a modificar suas estratégias de produção e compras de componentes. Uma forma de fazer isso foi a redução de preços pagos aos fornecedores e a instituição de programas de verificação da qualidade e sistemas para monitorar fornecedores, impactando diretamente as empresas de autopeças. Ainda segundo os autores, o complexo automotivo brasileiro passou a fazer parte destas transformações a partir dos anos 90, quando a economia do país foi liberalizada, e ficou em evidência a partir de 93 com a recuperação da economia e acordos entre montadoras, fornecedores e governo tornando o mercado automotivo brasileiro.

Atualmente o setor automotivo no Brasil, quarto maior do mundo, enfrenta grandes desafios. Com a desaceleração econômica, observa-se redução de vendas e aumento de dos estoques nas montadoras o que resultou em demissões em toda a cadeia de fornecimento.

\subsection{População e amostra}

A população deste estudo é constituída por 69 gestores de todos os departamentos da empresa Tower International, dos quais 41 concordaram em participar do processo e passaram a constituir a amostra desta pesquisa. Essa amostra de 41 gestores é apresentada na Tabela 1 de forma mais detalhada.

Tabela 1

Composição da Amostra por departamento com base em setembro 2011

\begin{tabular}{lccc}
\hline Departamento & Qtde & Respondentes & $\%$ \\
Produção & 11 & 2 & $18 \%$
\end{tabular}




\begin{tabular}{lccc} 
Engenharia de Desenvolvimento & 9 & 8 & $89 \%$ \\
Comercial & 6 & 6 & $100 \%$ \\
Engenharia Industrial & 6 & 5 & $83 \%$ \\
Manutenção & 6 & 4 & $67 \%$ \\
Qualidade & 6 & 3 & $50 \%$ \\
Finanças & 5 & 5 & $100 \%$ \\
Compras & 4 & 3 & $75 \%$ \\
Ferramentaria & 4 & 1 & $25 \%$ \\
Logística & 4 & 0 & $0 \%$ \\
Materiais & 3 & 1 & $33 \%$ \\
RH & 2 & 2 & $100 \%$ \\
TI & 2 & 1 & $50 \%$ \\
Segurança Patrimonial & 1 & 0 & $0 \%$ \\
\hline Total & $\mathbf{6 9}$ & $\mathbf{4 1}$ & $\mathbf{5 9 \%}$ \\
\hline Nota. Font: Dados pesquis
\end{tabular}

Nota. Fonte: Dados da pesquisa.

\section{a. Procedimentos de coleta de dados: instrumento e técnica}

Os dados foram coletados por meio de um procedimento de duas fases.

Fase 1.

Os participantes responderam a um instrumento de orientação social de valor, de acordo com Van Lange et al. (1997).

Esse instrumento teve por objetivo dividir a amostra em três grupos distintos, de acordo com sua orientação de valor social: pró-sociais, individualistas e competidores. Salienta Upton (2009) que esse instrumento tem excelentes qualidades psicrométricas e é internamente consistente, confiável e estável, fundamentado em diversos estudos.

O instrumento consiste em uma série de nove opções entre três pares de valores para o resultado próprio e resultado de outros. Cada um dos três pares de valores representa uma das três orientações de valores sociais (pró-social, individualista, concorrente). O item 1, v.g., no instrumento Social Values Orientation, tem as escolhas de A (480 pontos para si próprio e 80 para o outro), B (540 pontos para si próprio e 280 pontos para o outro) e C (480 pontos para si próprio e 480 pontos para o outro). Nessa escolha, o item A maximiza a vantagem relativa para si mesmo e representa opção de competidor; a escolha B maximiza o resultado para si mesmo e representa a opção individualista; e a escolha C maximiza o resultado das articulações e minimiza a diferença entre os resultados para si e para outros, e representa a opção pró-social. 
Para ser classificado como um dos tipos SVO, os participantes deveriam selecionar os pares de valores compatíveis com esse tipo SVO pelo menos em seis vezes das nove possíveis. Os participantes que não atenderam a esse critério foram classificados como "inconsistentes" e foram analisados juntamente com os competidores, conforme Upton (2009).

\section{Fase 2}

$\mathrm{Na}$ segunda fase do procedimento, os participantes foram submetidos a um experimento orçamentário a fim de se analisar o processo de formação de folga orçamentária (programa desenvolvido em computador). O cenário do experimento foi baseado em Stevens (2000) e consiste em submeter os participantes a uma tarefa de produção. Os participantes passaram por duas sessões de treinamento e, posteriormente, por três sessões de produção na qual determinaram sua melhor estimativa acerca das quantidades produzidas bem como o budget, o qual estará relacionado com o esquema de remuneração da empresa (fixo + bônus para cada unidade produzida acima do budget). Essas informações serviram de proxy para a determinação da variável de folga orçamentária.

Os procedimentos de coleta de dados estão resumidos na Tabela 2.

Tabela 2

Resumo dos procedimentos de coleta de dados com base em setembro 2011

\begin{tabular}{|c|c|c|c|c|}
\hline \# & Técnica & Modelo & Detalhes & Data \\
\hline 1 & Questionário & $\begin{array}{l}\text { Van Lange } \\
\text { et al. (1997) }\end{array}$ & $\begin{array}{l}\text { Instrumento para definir a orientação de valor social } \\
\text { (SVO) dos participantes. Consiste na escolha de pares } \\
\text { de valores para o resultado próprio e do parceiro e } \\
\text { partir do resultado, os indivíduos são classificados } \\
\text { entre: pró-sociais, individualistas ou competidores. }\end{array}$ & $\begin{array}{c}\text { Dados coletados } \\
\text { pessoalmente entre } \\
25 / 09 / 11 \text { a } \\
05 / 11 / 2011\end{array}$ \\
\hline 2 & Experimento & $\begin{array}{c}\text { Stevens } \\
(2000)\end{array}$ & $\begin{array}{l}\text { Experimento em computador que simula sessões de } \\
\text { uma tarefa de produção onde os participantes } \\
\text { determinam estimativas de produção e budget. O } \\
\text { esquema de compensação é formado por um salário } \\
\text { fixo + bônus para cada unidade produzida acima do } \\
\text { budget. }\end{array}$ & $\begin{array}{c}\text { Dados coletados por } \\
\text { email entre } 07 / 11 / 11 \mathrm{a} \\
13 / 12 / 2011\end{array}$ \\
\hline
\end{tabular}

Nota. As telas do experimento e questionário não foram incluídas no texto por falta de espaço, mas estão disponíveis com o primeiro autor. Fonte: Dados da pesquisa. .

\section{Apresentação e Discussão dos Resultados}

Os participantes foram distribuídos segundo o perfil SVO e de onde se obteve a distribuição apresentada na Tabela 3. 
Tabela 3

Distribuição da amostra de acordo com orientação de valor social com base em Setembro 2011

\begin{tabular}{cccccc}
\hline SVO & Qtde & $\%$ & SVO & Qtde & $\%$ \\
Cooperativos & 23 & $56 \%$ & Cooperativos & 23 & $56 \%$ \\
Individualistas & 12 & $29 \%$ & Individualistas & 12 & $29 \%$ \\
Competidores & 2 & $5 \%$ & Comp./Mix & 6 & $15 \%$ \\
Inconsistentes & 4 & $10 \%$ & & 41 & $100 \%$ \\
Total & 41 & $100 \%$ & Total & \\
& & \multicolumn{5}{c}{ Distribuição analisada } \\
\hline \multicolumn{2}{l}{ Distribuição dos respondentes }
\end{tabular}

Nota. Fonte: Dados da pesquisa.

Também foram realizados testes estatísticos para evidenciar a existência de folga orçamentária e relacioná-la com a orientação de valor social dos respondentes:

Tabela 4

Resultados dos dados com base em setembro 2011

\begin{tabular}{lccc}
\hline & BUDGET & PRODUÇÃO & FOLGA \\
\hline média & 9,732 & 10,862 & 1,13 \\
desvio-padrão & 3,272 & 3,667 & 2,08 \\
\hline $\mathrm{n}$ & 41 & 41 & 41 \\
\hline
\end{tabular}

Nota. Fonte:Dados da pesquisa.

Em primeiro lugar, foi realizado o comparativo entre budget e produção. Na tabela 4, os resultados demonstram que a média do budget é menor do que a produção, para ratificação deste resultado, foi calculado o teste $t$ pareado. O teste verificou se a média de budget é menor do que a da produção.

Tabela 5

Teste Pareado dos dados

Paired Samples Test

Mean $\quad$ Std. Deviation $\quad \begin{array}{cc}\text { Std. Error } \\ \text { Mean }\end{array} \quad \begin{gathered}95 \% \text { Confidence } \\ \text { Interval of the } \\ \text { difference }\end{gathered}$

ReFAE - Revista da Faculdade de Administração e Economia, v. 9, n. 1, p. 117-134, 2018 
Pair 1

Budget -

Produção $-1,12976$ $\begin{array}{lllllll}2,08126 & 0,32504 & -1,78668 & -0,47283 & 3,476 & 40\end{array}$

df Sig. (2-tailed)

Nota. Fonte: Dados da pesquisa.

De acordo com os resultados, também pôde-se concluir que existe evidência estatística indicando que a média de budget é menor que da Produção ( $\mathrm{p}=0,001<0,05$ ), o que configura constituição de Folgas Orçamentárias, confirmando a Hipótese H1.

Em segundo lugar, foi realizado o teste de análise de variância (Anova one way) para verificar se existe diferença na média do budget, da produção e da folga nos grupos: cooperativos, individualistas e competidores.

Tabela 6

\section{Análise de budget nos grupos ANOVA}

\begin{tabular}{cccccc}
\hline & Sum of Squares & DF & Mean Square & F & Sig. \\
Between Groups & 4,361 & 2 & 2,181 & 0,195 & 0,823 \\
Within Groups & 423,866 & 38 & 11,154 & & \\
Total & 428,227 & 40 & & & \\
\hline
\end{tabular}

Nota. Fonte: Dados da pesquisa.

Analisando-se o teste acima, pôde-se concluir que não existe diferença significativa entre os grupos $(\mathrm{p}=0,823)$ no tocante ao orçamento.

O mesmo procedimento foi feito em relação à produção

Tabela 7.

Análise de produção nos grupos ANOVA

\begin{tabular}{cccccc}
\hline & Sum of Squares & df & Mean Square & F & Sig. \\
Between Groups & 11,902 & 2 & 5,951 & 0,43 & 0,654 \\
Within Groups & 526,241 & 38 & 13,848 & & \\
Total & 538,142 & 40 & & & \\
\hline
\end{tabular}

Nota. Fonte: Dados da pesquisa.

Com base nos dados acima, constatou-se que não existe diferença significativa entre os grupos $(\mathrm{p}=0,654)$.

ReFAE - Revista da Faculdade de Administração e Economia, v. 9, n. 1, p. 117-134, 2018 
Por último, repetiu-se mais uma vez o procedimento em relação à folga orçamentária.

Tabela 8

Análise de folga nos grupos ANOVA

\begin{tabular}{lrrrrr}
\hline & Sum of Squares & DF & Mean Square & F & Sig. \\
\hline $\begin{array}{l}\text { Between } \\
\text { Groups }\end{array}$ & 1,88 & 2 & 0,94 & 0,209 & 0,813 \\
\hline Within Groups & 171,208 & 38 & 4,505 & & \\
\hline Total & 173,088 & 40 & & & \\
\hline
\end{tabular}

Nota. Fonte: Dados da pesquisa.

Com base na Tabela 8 , pode-se concluir que também não existe diferença significativa entre os grupos $(\mathrm{p}=0,813)$.

Dessa forma, com base nos resultados estatísticos, não foi possível validar a hipótese H2 de que diferentes tipos de orientação de valor social influenciam de forma diferenciada a formação de folga orçamentária.

Esses resultados apresentados não são consistentes com a teoria, o que pode sugerir que a constituição das Folgas Orçamentárias pode ser mais influenciada pelas características organizacionais do que pelas características pessoais. Os resultados desta pesquisa podem ser justificados por características peculiares ao contexto em que a Tower International enfrentava, durante o período de pesquisa, ou seja, pela realização do processo orçamentário de 2012, com alta pressão por redução de custos decorrentes de quedas nas vendas provocadas pelo aumento da concorrência de importados no mercado automotivo e pelo desastre ambiental no Japão, que prejudicou a produção de um de seus maiores clientes. Em outras palavras, os respondentes poderiam estar influenciados por esse ambiente e por um desejo de cumprir os objetivos orçamentários.

Outra possível explicação pode ser encontrada na literatura que diz que os valores pessoais são deixados de lado, ou seja, se a prática de constituição de folgas orçamentárias for feita pela maioria dos gestores, indivíduos com a necessidade de serem aceitos pelo grupo também o farão, mesmo que isso seja contrário aos seus valores pessoais. Stompka (1999) discorre sobre esse tema argumentando que diversos estudos em sociologia afirmam que a as normas sociais ou do grupo afetam as escolhas individuais. Valores individuais mudam na medida em que as pessoas convivem com grupos sociais com diferentes visões (Rohan, 2000). 


\section{Considerações Finais}

Este estudo analisou a relação entre a orientação de valor social e a formação de folga orçamentária. Com base nos resultados estatísticos obtidos, não foi possível suportar as hipóteses teóricas de que a orientação de valor social cooperativa contribui para a redução da folga orçamentária.

Vale ressaltar que este estudo possui algumas limitações. Primeiramente, considera apenas uma filial (Arujá) da empresa (Tower International). Em segundo lugar, os resultados podem sofrer influência em função do tamanho da amostra: 41 líderes participantes. Dessa forma, os resultados desta pesquisa não podem ser generalizados para outras empresas.

Finalmente, espera-se que esta pesquisa sirva de inspiração para trabalhos futuros, dada a importância do desempenho dos grupos de funcionários para o resultado das empresas. A percepção de objetivos de forma cooperativa pode contribuir para as práticas de contabilidade gerencial e para estudos sobre busca por competitividade organizacional.

Assim, esses resultados poderiam ser considerados pela Tower Automotive de modo a adequar seus processos e controles ao perfil de seus gestores e aos objetivos organizacionais, com vistas a potencializar o alinhamento de metas, estratégia e resultados organizacionais. Por exemplo, o processo orçamentário poderia ser conduzido de forma a aumentar a percepção de interdependência dos objetivos; as metas poderiam ser atribuídas aos departamentos de forma conjunta; os líderes poderiam estimular a resolução de conflitos de forma construtiva; a avaliação de desempenho poderia considerar o trabalho e resultado em equipe; a avaliação das variações entre realizado versus orçado poderia ser feita de forma a desestimular a formação de folga, assim a empresa poderia promover trabalhos para melhorar a aceitação do processo orçamentário de forma a aumentar o comprometimento, a cooperação entre funcionários e espírito de unidade.

\section{Referências}

BEUREN, I.M.; BECK, F.; Popik, F. (2014). Interesses Compartilhados Afetam a Veracidade dos Orçamentos? R. Cont. Fin. - USP, São Paulo, v. 26, n. 67, p. 11-26

BRIEF, A. P.; MOTOWIDLO, S. J. (1986). Prosocial organizational behaviors. The Academy of Management Review, 11(4), 710-725. doi: 10.5465/AMR.1986.4283909 
COSTA, I.; QUEIROZ, S. R. R. (2000). Autopeças no Brasil: mudanças e competitividade na década de 90. Revista de Administração, 35(3), 27-37.

CYERT, R. M.; MARCH, J. G. (1992). A behavioral theory of the firm (2a ed). Cambridge, Mass., USA: Blackwell Business, 268 p.

DIEHL, A. A.; TATIM, D. C. (2004). Pesquisas em ciências sociais aplicadas: Métodos e técnicas. São Paulo: Pearson Prentice Hall, 168 .

EISENHARDT, K. M. (1989). Agency theory: an assessment and review. The Academy Of Management Review, 14(1), 57-74. doi: 10.5465/AMR.1989.4279003

ETHERINGTON, L.; TJOSVOLD, D. (1998). Managing budget conflicts: contribution of goal interdependence and interaction. Canadian Journal of administrative sciences, 15(2), 142-151. doi:10.1111/j.1936-4490.1998.tb00158.x

FREZATTI, F.; RELVAS, T. R. S.; NASCIMENTO, A. R.; JUNQUEIRA, E.; OYADOMARI, J. C. T. (2010). Críticas ao orçamento: problemas com o artefato ou a não utilização de uma abordagem abrangente de análise? Advances in Scientific and Applied Accounting, 3(2), 190-216. Recuperado de http://asaaccounting.info/asaa/article/view/11/17

Henttu-AHO, T., \& JÄRVINEN, J. (2013). A field study of the emerging practice of beyond budgeting in industrial companies: an institutional perspective. European Accounting Review, 22(4), 765-785

HOPE, J.; FRASER, R. (2003). Who needs budgets? Harvard Business Review, 02/2003.

IWAI, T. (2014). Orientação de Valor Social, Sistema de Valores e Comportamento Cooperativo: Um Estudo Experimental. XXXVIII Encontro da ANPAD Rio de Janeiro/RJ - 13 a 17 de setembro de 2014

JUNQUEIRA, E.; OYADOMARI, J. C. T.; MORAES, R. (2010). Reservas orçamentárias: um ensaio sobre os fatores que levam à sua constituição. ConTexto, 10(17), 31-42. Recuperado de http://seer.ufrgs.br/index.php/ConTexto/article/view/14715/8613

LIBBY, T., \& LINDSAY, R. M. (2010). Beyond budgeting or budgeting reconsidered? A survey of North-American budgeting practice. Management Accounting Research, 21(1), 5675.

MERCHANT, K. A. (1981). The design of the corporate budgeting system: influences on managerial behavior and performance. The Accounting Review, 56(4), 813-829.

MERCHANT, K. A. (1985). Budgeting and the propensity to create budgetary slack. Accounting, Organizations and Society, 10(2), 201-210.

MERCHANT, K. A. (1990). The effects of financial controls on data manipulation and management myopia. Accounting, Organizations and Society, 15(4), 297-313.

MC CLINTOCK, C. G.; MESSICK, D. M. (1969). The definition, measurement and development of social motives underlying cooperative and competitive behavior. Washington (D.C.): Clearinghouse. 
OLIVEIRA, C. R. de; LAVARDA, C. E.; PATON, C. (2010, setembro) Fatores determinantes da folga orçamentária associados ao ciclo de vida organizacional. SEMINÁRIOS EM ADMINISTRAÇÃO DA FEA-USP (SEMEAD), 2010, SÃ̃O PAULO. XIII SEMEAD Sustentabilidade Ambiental nas Organizações. São Paulo, SP, Brasil, 13.

ROHAN, M. J. (2000). A rose by any name? The values construct. Personality and Social Psychology Review, 4(3), 255-277. doi: 10.1207/S15327957PSPR0403_4

SANTOS, M. F.; LUCIANETTI, L.; BOURNE, M. (2012). Contemporary performance measurement systems: A review of their consequences and a framework for research. Management Accounting Research 23 (2012) 79- 119

SCHWARTZ, S. T.; WALLIN, D. E.; YOUNG, R. A. (2007). Economic experiments for the management accounting classroom. Issues in Accounting Education, 22(3), 515-534. doi: 10.2308/iace.2007.22.3.515

STEVENS, D. (2000) Determinants of budgetary slack in the laboratory: an investigation of controls for self-interested behavior. Social Science Research Network disponível em: $\underline{\text { http://ssrn.com/abstract=219131 ou http://dx.doi.org/10.2139/ssrn.219131 }}$

SZTOMPKA, P. (1999). Trust: a sociological theory. Cambridge: Cambridge University Press, $228 \mathrm{p}$.

UPTON, D. R. (2009). Implication of social value orientation and budget levels on group performance and performance variance. Journal of Management Accounting Research, 21, 293-316. doi: 10.2308/jmar.2009.21.1.293

VAN DER STEDE, W. A. (2000) The relationship between two consequences of budgetary controls: budgetary slack creation and managerial short-term orientation. Accounting, Organizations and Society, 25(6), 609-622. doi: 10.1016/S0361-3682(99)00058-6

VAN LANGE, P. A. M; OTTEN, W.; DE BRUIN, E. M. N.; JOIREMAN, J. A. (1997). Development of prosocial, individualistic, and competitive orientations: theory and preliminary evidence. Journal of Personality and Social Psychology, 73(4), 733-46. doi: 10.1037//0022-3514.73.4.733 